\title{
Post-surgical evisceration with or without autocannibalism in 333 dogs-a survey of veterinarians
}

\author{
Mark Rishniw $^{1^{*}}$ and Lori R. Kogan ${ }^{2}$ \\ ${ }^{1}$ Veterinary Information Network, Davis, CA, USA \\ ${ }^{2}$ Clinical Sciences Department, College of Veterinary Medicine and Biomedical Sciences, Colorado State University, \\ Fort Collins, CO, USA
}

\begin{abstract}
Background: Few reports of post-surgical evisceration, with or without autocannibalism, in dogs exist.

Aims: To collect a large case series of dogs experiencing post-surgical evisceration, with or without autocannibalism. Methods: We surveyed practicing veterinarians who were members of the Veterinary Information Network about their experiences with post-surgical evisceration in dogs, variably accompanied by autocannibalism (ingestion of eviscerated organs or tissues). Responses were analyzed using descriptive statistics.

Results: We identified 333 cases with sufficient information for analysis: 221 with evisceration and autocannibalism, and 112 with just evisceration. Most cases occurred following surgery for reproductive reasons (desexing, cesarean section, and pyometra). Most occurred in young adult or adult dogs. Most dogs received analgesia perioperatively had routine closure (simple interrupted or simple continuous muscle layer closure) and most did not wear an E-collar post-surgically. Most dogs eviscerated within 3 days of the initial surgery. Approximately $64 \%$ underwent surgical repair and survived long-term without complications, more frequently if the evisceration was not accompanied by autocannibalism.
\end{abstract}

Conclusion: Our study suggests that post-surgical evisceration and autocannibalism can generally be successfully managed by practitioners and do not confer a uniformly poor outcome for the dog.

Keywords: Dehiscence, Eventration, Ovariohysterectomy, Surgery, Trauma.

\section{Introduction}

Dogs, and occasionally cats, can suffer a breakdown of sutures that close a laparotomy, resulting in the evisceration of abdominal viscera (Gower et al., 2009). However, descriptions of circumstances surrounding these events are limited to a single case series of 12 animals. Completely undescribed is the phenomenon of subsequent autocannibalism of the abdominal viscera-essentially, eating one's own eviscerated organs. One of the authors' personal experience with such an event and discussion with colleagues prompted this study, designed to determine whether this event is as rare as initially suspected, and what outcomes might be expected for dogs presenting with post-surgical evisceration, with or without autocannibalism.

Therefore, we created a survey to obtain additional information about such events and distributed it to veterinarians who are members of the Veterinary Information Network (VIN). We hypothesized that outcomes for dogs presenting with eviscerated (and ingested) abdominal viscera following routine laparotomy would have a reasonable clinical outcome in most cases.

\section{Materials and Methods}

We used an online survey system to gather recollections about dogs that had presented to veterinarians with abdominal evisceration, with or without autocannibalism. The survey was distributed by email on October 31st, 2018 to all veterinarians who were members of VIN, and remained open for responses from October 31st to November 15th, 2018. A single reminder email was sent on November 7th, 2018. No ethical review was sought or obtained as this was an anonymous survey.

The survey asked respondents if they had ever been presented with a case of evisceration \pm autocannibalism in a dog that had recently undergone a surgical laparotomy, and if they had been presented with a case, whether they had observed more than one case. For respondents answering "Yes", they were asked a series of questions regarding the event(s). These included:

(A) Whether the dog was a purebred (and if so, what breed)

(B) Age of dog: puppy ( $<6$ months), immature adult ( 6 months to $<1$ year of age), adult (1-9 years), or geriatric ( $>9$ years)

(C) Why the original laparotomy was performed

(D) Whether or not the dog received perioperative analgesia

(E) What abdominal closure pattern the original surgeon used 
(F) Whether or not the dog was wearing an Elizabethan collar at the time of evisceration

(G) Whether the dog was a pet, shelter animal, or other

(H) The ultimate outcome of the case

(I) Reporting clinician's type of practice when presented with the eviscerated case

Because most of the survey relied on respondents' recall, often without the benefit of medical records (due to changing of employment, retirement, etc.), we did not ask specific details about the types of repair, complications, or dates when these cases presented. We analyzed most of the data using descriptive statistics. We used Chi-square tests of independence to compare the ultimate outcomes of dogs suffering just evisceration with those that experienced evisceration with autocannibalism.

\section{Results}

The survey was distributed to 38,531 members of VIN. Five-hundred and twenty-five veterinarians completed the survey. Of these, 233 (44\%) reported never encountering a case of post-surgical evisceration; $260(50 \%)$ reported seeing one case, 23 (4\%) reported seeing two cases, and nine (2\%) reported seeing three cases, for a total of 333 cases. Veterinarians reported on 221 cases $(66 \%)$ where dogs both eviscerated and cannibalized their viscera, and 112 cases (34\%) where dogs only suffered evisceration. Most respondents $(90 \%)$ were working either in general practice or emergency practice when presented with their evisceration case(s). One hundred and forty-two dogs (43\%) were mixed breed and 151 were purebred (45\%). In 40 cases, respondents could not recall the breed. Breeds represented by more than five dogs included: Labrador retriever (28), German Shepherd (11), Chow Chow (8), Dachshund (7), and Siberian Husky (7). Of the 333 cases, 27 (8\%) were puppies, $169(51 \%)$ were young adults, $117(35 \%)$ were adults, $5(2 \%)$ were geriatric; in 11 cases $(3 \%)$, respondents did not recall the age and four respondents (1\%) provided no response. Reasons for the initial laparotomy included desexing (261 dogs, 78\%), foreign body removal (20 dogs, 6\%), exploratory surgery (16 dogs, 5\%), caesarian section (14 dogs, 4\%), trauma (4 dogs, 1\%), prophylactic gastropexy (3 dogs, 1\%); 10 respondents (3\%) could not recall, and 1 respondent provided no response.

Veterinarians reported providing pre- and postoperative analgesia in 154 cases $(68 \%)$, pre-operative analgesia only in 30 cases (13\%), post-operative analgesia in 17 cases (7\%), and no analgesia in 26 cases (11\%); in 103 cases (31\%), respondents could not recall analgesic protocols and three respondents provided no responses. The initial laparotomy was closed using simple interrupted sutures in 111 cases (48\%), simple continuous pattern in 104 cases $(45 \%)$, cruciate or mattress patterns in 8 cases (4\%), Ford interlocking pattern in 2 cases ( $1 \%)$, and "other" patterns in 5 cases (2\%); 100 respondents could not recall the suture pattern used, and three respondents failed to provide a response.

In 262 cases, respondents reported the time (in days) after the initial laparotomy that transpired before the evisceration; of these, 189 (72\%) occurred within 3 days, $21 \%$ occurred between 3 and 7 days, and only $7 \%$ occurred after the first week. Of the 333 cases, 267 (91\%) were not wearing an E-collar at the time of the event, $27(9 \%)$ were, and 39 respondents could not recall details.

Of the 333 cases, 238 (78\%) were considered by the respondent to be "established family pets", 39 (13\%) were recently obtained pets, $26(9 \%)$ were dogs in a shelter situation, and 30 respondents could not recall. Outcomes were reported in 320/333 cases. Of all reported cases, 205 (64\%) survived long-term. However, outcomes differed between dogs suffering only evisceration and dogs that eviscerated and then ate their viscera (Table 1). Specifically, more dogs suffering only evisceration survived long-term than dogs that ate their viscera $(76 \%$ vs. $58 \%, p=0.006)$. Similarly, fewer dogs that only eviscerated were euthanized without surgery $(16 \% v s .26 \%, p=0.048)$.

\section{Discussion}

Our study provides clinically relevant information on a large cohort of dogs suffering post-surgical evisceration with or without autocannibalism. Importantly, most dogs suffering such trauma, if repaired, had good longterm outcomes. However, those that did not eat their own viscera had better outcomes than those that did. Our data expand considerably on the previous study by Gower and colleagues, who reported on 12 cases of evisceration (Gower et al., 2009). In that study, all dogs survived. However, all the dogs in that study had presented to a university hospital and received specialist care. On the other hand, most of the respondents in our study were either general practitioners or working in an emergency clinic when presented with their cases. Therefore, our outcome data are more generalizable to veterinary clinicians in first opinion or emergency practice. Furthermore, none of the dogs in the previous study engaged in autocannibalism. Our study suggests that the ingestion of viscera results in worse outcomes. However, some of this is attributable to the higher proportion of these dogs that were euthanized without attempted repair. We do not know whether this was because the visceral trauma was deemed irreparable and fatal, or because the clinician opted not to attempt repair for other reasons (e.g., fear, inexperience, cost, etc.). Although Gower and colleagues did not report on cases of autocannibalism, four of the animals in their study required resection of compromised intestines; all four survived to discharge (Gower et al., 2009). Similarly, Gorman et al. (2006) reported on extensive 
Table 1. Comparison of outcomes for 320 dogs that eviscerated with and without subsequent autocannibalism.

\begin{tabular}{lcc}
\hline Outcome & $\begin{array}{c}\text { Evisceration only } \\
(\boldsymbol{n}=\mathbf{1 0 6})\end{array}$ & $\begin{array}{c}\text { Evisceration and autocannibalism } \\
(\boldsymbol{n}=\mathbf{2 1 4})\end{array}$ \\
\hline Surgery with complete recovery & $80(75 \%)^{*}$ & $103(48 \%)$ \\
Surgery with partial recovery & $1(1 \%)^{*}$ & $21(10 \%)$ \\
Post-operative death & $4(4 \%)$ & $29(14 \%)$ \\
Euthanasia without surgery & $17(16 \%) \dagger$ & $55(26 \%) \dagger$ \\
Death prior to presentation & $4(4 \%)$ & $5(2 \%)$ \\
\hline
\end{tabular}

$(*)$ : More dogs survived long-term after evisceration only than after both evisceration and autocannibalism $(p=0.006)$.

$(\dagger)$ : Fewer dogs that eviscerated only were euthanized without attempting surgical repair than dogs that eviscerated with subsequent autocannibalism $(p=0.048)$

intestinal resection in 13 dogs, most of which had a good outcome. Interestingly, in that study, the authors found no association between the amount of intestine resected and the outcome. Our data, along with outcomes of these previous studies, should encourage clinicians to attempt surgical repair, even if the dog has ingested some of its viscera. Our data expand on those of older, previous studies of post-ovariohysterectomy complications, where authors did not report any evisceration or autocannibalism events in a combined total of 214 procedures (Burrow et al., 2005; Pearson, 1973).

Most dogs that eviscerated were not wearing E-collars at the time of the event. However, this is not surprising, as E-collars are not routinely applied to dogs undergoing routine abdominal surgery. Given the relatively infrequent nature of these events, we cannot recommend that E-collars become "standard of care" for abdominal surgeries.

Most dogs that eviscerated did so soon after surgery. The circumstances that lead to the event remain unknown. We presumed that these dogs suffered wound dehiscence secondary to self-mutilation or self-trauma, but have no proof of this. Most dogs in our study were either young adults or adults. Only two geriatric dogs undergoing laparotomy for non-reproductive reasons ( $2 \%$ of all dogs undergoing such procedures) opened their abdominal incisions. The other three geriatric dogs had undergone surgery for pyometra $(1 \%$ of all dogs undergoing reproductive surgery). Therefore, if clinicians are concerned about potential evisceration, attention should be concentrated on the immediate post-operative period (first 3-4 days), mostly in adult, non-geriatric dogs.

Clinicians reported certain breeds more frequently than others. Labradors and possibly German Shepherds appear to be over-represented; however, the popularity of these breeds makes any true estimates uninterpretable. On the other hand, Chow Chows, Siberian Huskies, and Dachshunds are not nearly as popular. We were surprised to see multiple reports of post-surgical evisceration in these breeds, but prefer not to speculate about possible reasons.

Our study has the usual limitations of survey data. The respondents provided information mostly from memory (we did not require them to consult medical records). Consequently, information about suture patterns, analgesia, etc., should be interpreted cautiously, because these might be based on "what we did with all cases at that time in that practice." Indeed, such information is often omitted from medical records. Furthermore, we cannot provide true prevalence estimates, because the sample is a convenience sample, rather than a true cross-sectional study. Nevertheless, as a large case series, our data still provide useful clinical information about a relatively uncommon event.

\section{Conclusion}

Dogs that suffer post-surgical evisceration, with or without subsequent autocannibalism have a reasonable prognosis if the trauma is repaired surgically. Clinicians should, in most cases, attempt such repair unless they can confirm that the trauma is fatally extensive.

\section{Acknowledgments}

This research did not receive any specific grant from funding agencies in the public, commercial, or notfor-profit sectors. We would like to thank the member veterinarians of the Veterinary Information Network for participating in the survey.

\section{Conflict of interest}

One of the authors is an employee of the Veterinary Information Network.

\section{Authors' contributions}

Mark Rishniw co-designed the survey, performed the analyses, and drafted the manuscript. Lori Kogan co-designed the survey, edited, and approved the manuscript drafts.

\section{Ethical approval}

We did not seek ethical approval as the study was a survey of veterinary experiences and involved no animals. Respondents provided all responses voluntarily, without prejudice or compensation. 


\section{References}

Burrow, R., Batchelor, D. and Cripps, P. 2005. Complications observed during and after ovariohysterectomy of 142 bitches at a veterinary teaching hospital. Vet. Rec. 157, 829-833.

Gorman, S.C., Freeman, L.M., Mitchell, S.L. and Chan, D.L. 2006. Extensive small bowel resection in dogs and cats: 20 cases (1998-2004). J. Am. Vet. Med. Assoc. 228, 403-407.
Gower, S.B., Weisse, C.W. and Brown, D.C. 2009. Major abdominal evisceration injuries in dogs and cats: 12 cases (1998-2008). J. Am. Vet. Med. Assoc. 234, 1566-1572.

Pearson, H. 1973. The complications of ovariohysterectomy in the bitch. J. Small Anim. Pract. 14, 257-266. 